\title{
Double-port measurements for robust quantum optical metrology
}

\author{
Wei Zhong, ${ }^{1,2}$ Lan Zhou, ${ }^{3}$ and Yu-Bo Sheng ${ }^{1, *}$ \\ ${ }^{1}$ Institute of Quantum Information and Technology, \\ Nanjing University of Posts and Telecommunications, Nanjing 210003, China \\ ${ }^{2}$ National Laboratory of Solid State Microstructures, Nanjing University, Nanjing 210093, China \\ ${ }^{3}$ School of Science, Nanjing University of Posts and Telecommunications, Nanjing 210003, China
}

\begin{abstract}
It has been proposed and demonstrated that path-entangled Fock states (PEFSs) are robust against photon loss over NOON states [S. D. Huver et al., Phys. Rev. A 78, 063828 (2008)]. However, the demonstration was based on a measurement scheme which was yet to be implemented in experiments. In this work, we quantitatively illustrate the advantage of PEFSs over NOON states in the presence of photon losses by analytically calculating the quantum Fisher information. To realize such an advantage in practice, we then investigate the achievable sensitivities by employing three types of feasible measurements: parity, photon-number-resolving, and homodyne measurements. We here apply a double-port measurement strategy where the photons at each output port of the interferometer are simultaneously detected with the aforementioned types of measurements.
\end{abstract}

\section{Introduction}

An essential task in quantum optical metrology is to reach the ultimate sensitivity limit to the phase measurement imposed by quantum theory [1-8]. To date, extensive strategies have been proposed to improve the sensitivities of phase measurements. Among these, one celebrated strategy is the use of NOON states [1]

$$
|N:: 0\rangle=\frac{1}{\sqrt{2}}(|N, 0\rangle+|0, N\rangle) .
$$

It has been experimentally demonstrated that this strategy can acquire a Heisenberg-scaling sensitivity in lossless optical interferometry [9-13], which is a $\sqrt{N}$ factor improvement over the shot-noise limit (SNL) [1-6]. However, such a quantum improvement will be completely gone even if an individual photon loss occurs [14-16].

To mitigate this problem, two main kinds of protocols have been raised: active and passive. The former tries to actively reduce the effects of losses by managing experimental processes, e.g., applying quantum error correction $[17,18]$. The latter tries to passively retain sub-SNL sensitivities by using certain probe states which are more robust against losses but at the expense of a bit of sensitivity [14, 19-24]. Among these passive protocols, one of the most representative proposed by Huver et al. is that of taking the path-entangled Fock states (PEFS) [19]

$$
|m:: n\rangle=\frac{1}{\sqrt{2}}(|m, n\rangle+|n, m\rangle), \quad(m>n)
$$

as the probe state. The authors in [19] demonstrated that the PEFSs show more robustness against photon loss than NOON states under the constraint of $\Delta \equiv m-n=N$ and referred to it as robust optical metrology. However, the demonstration was based on a measurement scheme

\footnotetext{
* shengyb@njupt.edu.cn
}

which was yet to be implemented. Later, Jiang et al. considered the single-parity (SP) measurement scheme, but failed to observe the robustness of the PEFS scheme compared to the NOON state scheme [24]. Whether or not the PEFSs are robust to loss over NOON states in practice is still an open question.

In this paper, we address this issue by finding effective measurements to present the robustness of the PEFS scheme in practical metrological experiments. To quantitatively demonstrate the advantage of the PEFS scheme, we first derive the lower sensitivity bounds in the presence of photon losses by invoking quantum Fisher information (QFI), since the QFI is a figure of merit to indicate the performance of a metrological scheme. We then discuss the achievable sensitivities with three different types of measurements: parity, photon-numberresolving, and homodyne measurements. All these measurements are achievable in today's state-of-the-art experiments. Here we employ a double-port measurement protocol, in which the photons at each output port of the interferometer are simultaneously detected with the three aforementioned types of measurements. The reason why we utilize this protocol comes from consideration of the superiority of the double-port measurement over the single-port one (see Appendix A for a demonstration of this superiority). Although all aforementioned measurements were systematically studied in lossless cases [25-29], there is little knowledge of the effect of these measurements in realistic experiments where losses are present. Our work serves to complement studies in this aspect. Thus it will provide valuable information on the choice of measurement scheme which is more adequately implemented in the laboratory.

This paper is organized as follows. In Sec. II we revisit the robust optical metrology with PEFSs proposed by Huver et al. [19] and derive the lower sensitivity bounds based on quantum estimation theory. In Sec. III we discuss the achievable sensitivities with three different types of measurement. A summary and our conclusions are given in Sec. IV. 


\section{Robust quantum-optical interferometry with PEFSs}

A general optical two-mode interferometer consists of three optical elements: two beam splitters (BSs) denoted by $\hat{B}$ and a phase shifting denoted by $\hat{U}$ (see Fig. 1 ). This device serves to illustrate the principle of phase estimation. The input light is divided into two beams through the first BS. Then they accumulate an unknown phase $\phi$ of interest under the phase shifting. The value of the phase parameter is finally read out by detecting the photons in the beams out from the second BS.

From quantum estimation theory [30-32], the sensitivity of estimating the phase $\phi$ is statistically measured by the unit-corrected mean-square deviation of the estimator $\phi_{\text {est }}$ from the true value $\phi$,

$$
\left(\delta \phi_{\mathrm{est}}\right)^{2}=\left\langle\left(\frac{\phi_{\mathrm{est}}}{\partial_{\phi}\left\langle\phi_{\mathrm{est}}\right\rangle_{\mathrm{av}}}-\phi\right)^{2}\right\rangle_{\mathrm{av}},
$$

where $\langle\cdot\rangle_{\text {av }}$ denotes the statistical average and the derivative $\partial_{\phi}\left\langle\phi_{\text {est }}\right\rangle_{\text {av }}$ removes the local difference in the "units" of $\phi_{\text {est }}$ and $\phi$. Whichever measurement scheme is employed, the ultimate limit to the sensitivity of the unbiased estimator is given by the quantum Cramér-Rao bound

$$
\left(\delta \phi_{\mathrm{est}}\right)^{2} \geq \frac{1}{v F_{Q}}
$$

where $v$ is the repetition of the experiment and $F_{Q}$ is the so-called QFI [30-32]. This bound is asymptotically achieved for large $v$ under optimal measurements, followed by the maximum likelihood estimator [30-32]. Although optimal measurements for saturating this bound have been formally demonstrated in Refs. [32, 33], they may be not achievable in realistic experiments.

Assuming that $a$ and $b$ denote the annihilation operators of upper and lower modes, respectively, we then define the Schwinger representation [34] as

$$
\begin{aligned}
& \hat{J}_{x}=\frac{1}{2}\left(a^{\dagger} b+a b^{\dagger}\right), \quad \hat{J}_{y}=\frac{1}{2 i}\left(a^{\dagger} b-a b^{\dagger}\right), \\
& \hat{J}_{z}=\frac{1}{2}\left(a^{\dagger} a-b^{\dagger} b\right),
\end{aligned}
$$

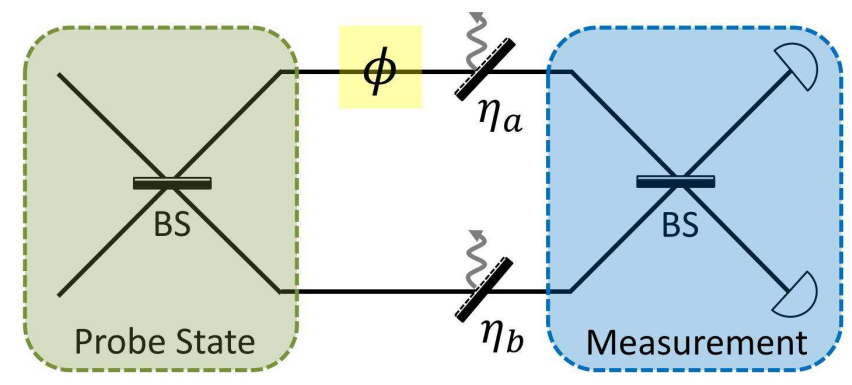

Figure 1. (Color online) Schematic of the optical two-arm interferometric setup in the presence of photon losses. which satisfy the commutation relations for the Lie algebra of $s u(2)$,

$$
\left[\hat{J}_{x}, \hat{J}_{y}\right]=i \hat{J}_{z}, \quad\left[\hat{J}_{y}, \hat{J}_{z}\right]=i \hat{J}_{x}, \quad\left[\hat{J}_{z}, \hat{J}_{x}\right]=i \hat{J}_{y} .
$$

and commute with the total photon number operator $\hat{N}=a^{\dagger} a+b^{\dagger} b$. For simplicity, we define $\hat{J}_{0}=\hat{N} / 2$ below. Within this representation, the operations of the BS and phase shifting are represented by

$$
\hat{B}=\exp \left(-i \frac{\pi}{2} \hat{J}_{y}\right), \quad \hat{U}=\exp \left(i \phi a^{\dagger} a\right)
$$

where $\hat{B}$ refers to a balanced $\mathrm{BS}$ and $\hat{U}$ accounts for the phase imprinting solely on mode $a$. An interferometry with asymmetric BSs has been discussed recently for various states $[35,36]$. As for phase shifting $\hat{U}$, another botharm configuration is represented by $\exp \left(i \phi \hat{J}_{z}\right)$, which has been widely considered in previous studies. Although these two types of phase shifting have a subtle difference in phase estimation [37], they have the same effect in our cases. This is because the states of consideration here are definite photon numbers and thus the phase shifts accumulating by single- and both-arm phase shifting are up to an irrelevant global phase factor, i.e., $\exp (i \phi N / 2)$.

In our work, as shown in Fig. 1, the probe state refers to the state after the first BS, i.e., $|\psi\rangle=\hat{B}\left|\psi_{\text {in }}\right\rangle$, where $\left|\psi_{\text {in }}\right\rangle$ is the input state powering at the input port of the interferometer. After the phase shifting $\hat{U}$, it becomes $|\psi(\phi)\rangle=\hat{U}|\psi\rangle$ acquiring an unknown phase to be estimated. Taking the NOON state of Eq. (1) as the probe state, one can acquire the Heisenberg-limit sensitivity associated with $F_{Q}=N^{2}$. However, in a practical situation, the interferometry is often subjected to photon losses. This may cause the dedicated NOON state to rapidly lose its quantum advantage for phase resolution. To circumvent this problem, Huver et al. proposed a robust metrological scheme by applying the PEFS as the probe state [19]. In the lossless case, the QFI for the PEFS of Eq. (2) reads $F_{Q}=\Delta^{2}$.

Below, we revisit this robust metrological scheme and derive the sensitivity bounds in the presence of photon losses by using the QFI. Here, photon losses are modeled by inserting fictitious BSs with transmissivities $\eta_{x}(x=a, b)$ into two arms of the interferometer [14, 19, 22]. Their effects can be formally represented in a Kraus form as

$$
\rho=\sum_{l_{a}, l_{b}=0}^{\infty} \hat{K}_{a, l_{a}} \hat{K}_{b, l_{b}} \rho_{\mathrm{in}} \hat{K}_{b, l_{b}}^{\dagger} \hat{K}_{a, l_{a}}^{\dagger}
$$

with Kraus operators

$$
\hat{K}_{x, l}=\left(1-\eta_{x}\right)^{l / 2}\left(\sqrt{\eta_{x}}\right)^{x^{\dagger} x} x^{l} / \sqrt{l !} .
$$

Due to the commutation relationship between phase shifting and photon losses [14, 22], one can freely exchange the order of operations between the phase shift and the photon loss. For simplicity, we assume that photon losses act after the phase shifting, as shown in Fig. 1. 
Successively going through the phase shifting and the photon losses, the probe state of Eq. (2) of $\Xi \equiv m+n$ evolves into a parametric mixed state as $[19,24]$

$$
\begin{aligned}
\rho(\phi)= & \frac{1}{2} \sum_{l_{a}=0}^{\Xi} \sum_{l_{b}=0}^{\Xi-l_{a}}\left[B_{l_{a} l_{b}}^{m} C_{l_{a} l_{b}}^{m}\left|m-l_{a}, n-l_{b}\right\rangle\left\langle m-l_{a}, n-l_{b}\right|\right. \\
& +B_{l_{a} l_{b}}^{n} C_{l_{a} l_{b}}^{n}\left|n-l_{a}, m-l_{b}\right\rangle\left\langle n-l_{a}, m-l_{b}\right| \\
& +\sqrt{B_{l_{a} l_{b}}^{m} B_{l_{a} l_{b}}^{n}} C_{l_{a} l_{b}}^{m} C_{l_{a} l_{b}}^{n} e^{i \Delta \varphi}\left|m-l_{a}, n-l_{b}\right\rangle \\
& \left.\times\left\langle n-l_{a}, m-l_{b}\right|+\text { H.c. }\right],
\end{aligned}
$$

with

$$
\begin{aligned}
& B_{l_{a} l_{b}}^{k} \equiv\left(\begin{array}{c}
k \\
l_{a}
\end{array}\right)\left(\begin{array}{c}
\Xi-k \\
l_{b}
\end{array}\right) \eta_{a}^{k}\left(\eta_{a}^{-1}-1\right)^{l_{a}} \eta_{b}^{\Xi-k}\left(\eta_{b}^{-1}-1\right)^{l_{b}}, \\
& C_{l_{a} l_{b}}^{k} \equiv H\left[k-l_{a}\right]-H\left[k-\Xi+l_{b}-1\right],
\end{aligned}
$$

where $(\bullet)$ denotes the binomial coefficient and $H[n]$ is the Heaviside step function of a discrete form. For NOON states, i.e., $m=N$ and $n=0$, Eq. (11) can be simplified as a direct sum form of

$$
\rho(\phi)=|\xi(\phi)\rangle\langle\xi(\phi)| \oplus \rho_{D},
$$

where the $\phi$-dependent state $|\xi(\phi)\rangle$ is given by

$$
|\xi(\phi)\rangle=\frac{1}{\sqrt{2}}\left(\sqrt{\eta_{a}^{N}} e^{i N \varphi}|N, 0\rangle+\sqrt{\eta_{b}^{N}}|0, N\rangle\right),
$$

up to a normalization constant and another part is a $\phi$ independent diagonal matrix of dimension $2 N$ as

$\rho_{D}=\frac{1}{2} \sum_{l=1}^{N}\left(B_{l 0}^{N}|N-l, 0\rangle\left\langle N-l, 0\left|+B_{0 l}^{0}\right| 0, N-l\right\rangle\langle 0, N-l|\right)$.

To calculate the QFI, one should determine the eigenvalues and eigenstates of $\rho(\phi)$ of Eq. (11). However, an analytical diagonalization of $\rho(\phi)$ may be not easily obtained in the case with photon losses in both beams. Following Ref. [14], we obtain the upper bound of the QFI for PEFSs, when losses occur in two arms, as

$F_{Q} \leq 2\left(m^{2}+n^{2}\right)-2 \sum_{l_{a}=0}^{\Xi} \sum_{l_{b}=0}^{\Xi-l_{a}} \frac{\left(m B_{l_{a} l_{b}}^{m} C_{l_{a} l_{b}}^{m}+n B_{l_{a} l_{b}}^{n} C_{l_{a} l_{b}}^{n}\right)^{2}}{B_{l_{a} l_{b}}^{m} C_{l_{a} l_{b}}^{m}+B_{l_{a} l_{b}}^{n} C_{l_{a} l_{b}}^{n}}$.

This inequality is saturated when losses occur only in one arm, i.e., $\eta_{b}=1$, in which case, Eq. (17) reduces to [14]

$$
F_{Q}=2\left(m^{2}+n^{2}\right)-2 \sum_{l_{a}=0}^{\Xi} \frac{\left(m B_{l_{a} 0}^{m} C_{l_{a} 0}^{m}+n B_{l_{a} 0}^{n} C_{l_{a} 0}^{n}\right)^{2}}{B_{l_{a} 0}^{m} C_{l_{a} 0}^{m}+B_{l_{a} 0}^{n} C_{l_{a} 0}^{n}} \text {. }
$$

In addition, it is also saturated for lossy NOON states of Eq. (14) as

$$
F_{Q}^{\mathrm{NOON}}=2 N^{2}\left(\frac{\eta_{a}^{N} \eta_{b}^{N}}{\eta_{a}^{N}+\eta_{b}^{N}}\right)
$$
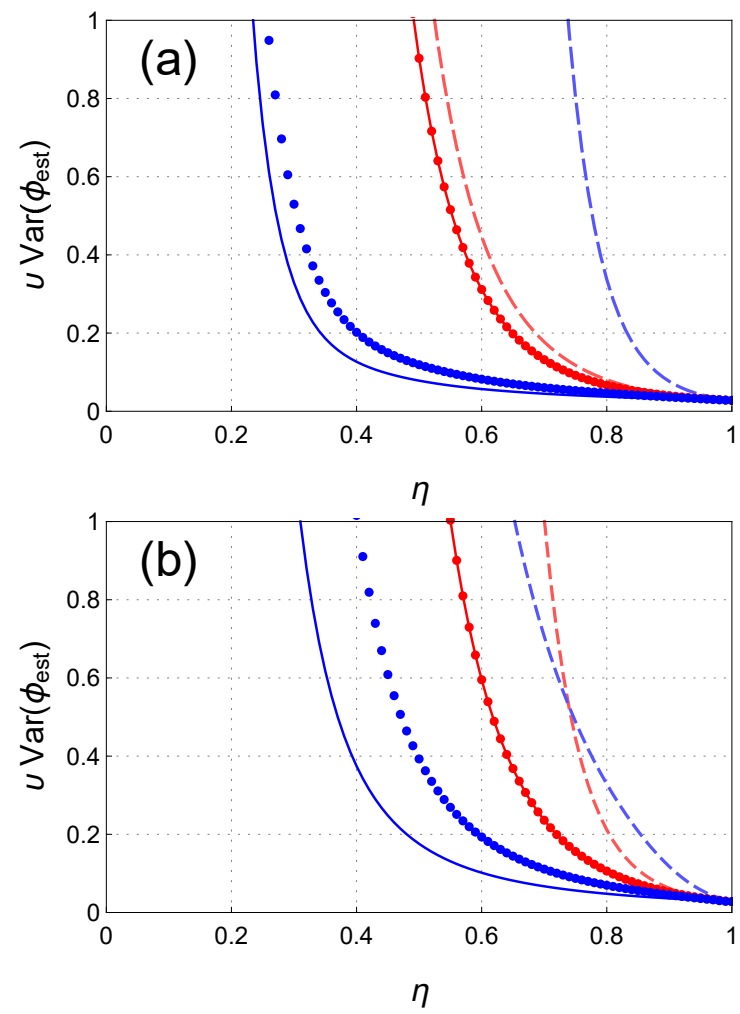

Figure 2. (Color online) Phase sensitivities versus transmissivity $\eta$ for losses in (a) both arms, i.e., $\eta_{a}=\eta_{b}=\eta$, and (b) one arm, i.e., $\eta_{a}=\eta$ and $\eta_{b}=1$. Different colors represent different probe states: Red is for $|6:: 0\rangle$ and blue is for $|10:: 4\rangle$. Solid lines correspond to the theoretical bounds of phase sensitivity, Dashed lines refer to the sensitivities obtained by the DP measurement and dotted lines to those by the DPNR measurement.

This can be easily obtained by employing the property that the QFI for a density matrix of direct sum form $\rho(\phi)=\bigoplus_{i=1}^{n} \rho_{i}(\phi)$ is given by the sum over all amounts of QFI in terms of each submatrices, i.e., $F_{Q}[\rho(\phi)]=$ $\sum_{i=1}^{n} F_{Q}\left[\rho_{i}(\phi)\right]$. For lossy NOON states, the $\rho_{D}$ of Eq. (16) does not contribute to the amount of QFI due to the fact that $\rho_{D}$ is independent of $\phi$. Hence the QFI for lossy NOON states is given by $F_{Q}=F_{Q}[|\xi(\phi)\rangle]$ and then derived as Eq. (19) with Eq. (15).

In order to compare the performance of the two strategies in lossy optical interferometry, we plot in Fig. 2 the sensitivity bounds for $|10:: 4\rangle$ and $|6:: 0\rangle$ according to Eqs. (17)-(19). It explicitly indicates that $|10:: 4\rangle$ shows more robustness to photon loss than $|6:: 0\rangle$. Although a similar conclusion was obtained by Huver et al. [19], it was based on a measurement scheme which was yet to be implemented. In what follows, we address how to realize this robustness in practice with feasible measurements. 

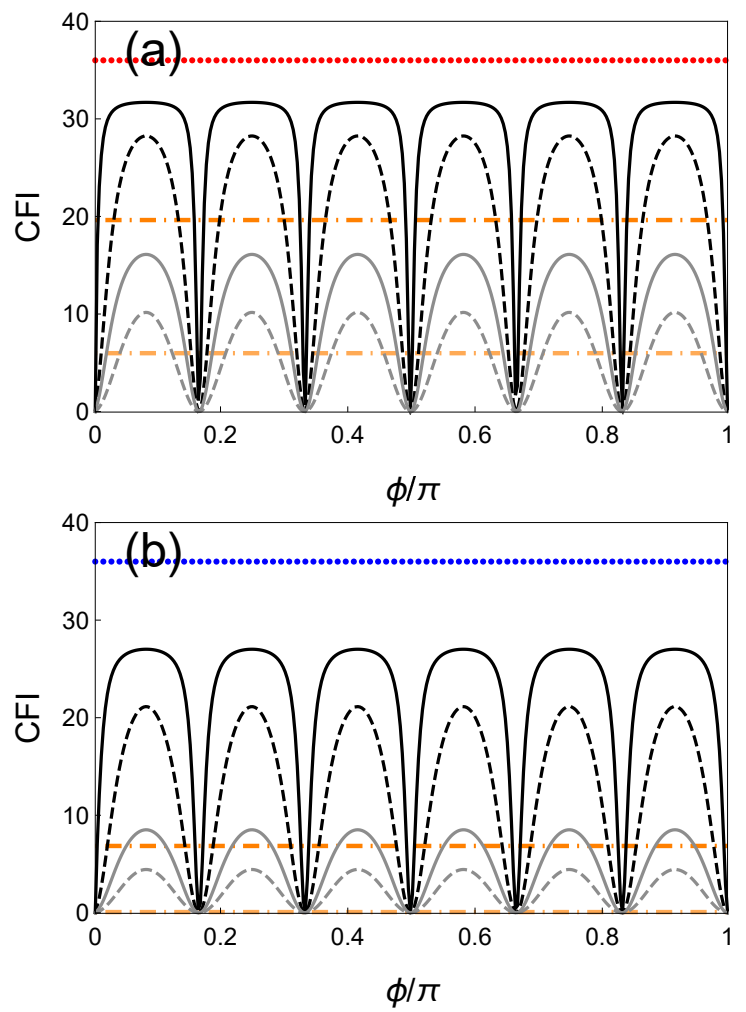

Figure 3. (Color online) Classical Fisher information as a function of $\phi$ for (a) $|6:: 0\rangle$ and (b) $|10:: 4\rangle$ with equal photon loss rates on each arm $\eta_{a}=\eta_{b}=\eta$. Dotted lines represent the lossless case, i.e., $\eta=1$. The upper darker lines for different colors and the lower lighter ones refer to the loss cases with $\eta=0.98$ and 0.9 , respectively. Solid, dashed and dash-dotted curves correspond to the DP, SP, and DH measurements, respectively.

\section{Phase sensitivities with three feasible measurements}

In quantum theory, a generic measurement can be described by a positive-operator-valued measurement $\hat{M} \equiv$ $\left\{\hat{M}_{\chi}\right\}$ with $\chi$ the results of the measurement. Given an operator $\hat{\boldsymbol{M}}$, the accessible phase sensitivity is limited by a classical analog of the inequality $(4)(\delta \hat{\phi})^{2} \geq\left(v F_{C}\right)^{-1}$, where $F_{C}$ is the classical Fisher information (CFI) defined as

$$
F_{C}=£ \frac{1}{p(\chi \mid \phi)}\left(\frac{d p(\chi \mid \phi)}{d \phi}\right)^{2} d \chi
$$

Here $p(\chi \mid \phi) \equiv \operatorname{Tr}\left[\hat{M}_{\chi} \rho(\phi)\right]$ represents the probability of the outcome $\chi$ with the specific value of $\phi$ and the sum or integral needs to be evaluated for discrete or continuous variables $\chi$. This bound is achievable for the maximum-likelihood estimator with the Bayesian estimation method when $v$ is sufficiently large [30, 31, 38, 39]. If the equality $F_{C}=F_{Q}$ holds, $\hat{M}$ is the optimal measurement. In lossless interferometry, it has been demonstrated that parity measurement [40], photon-number- resolving measurement $[26,27,41]$, and homodyne measurement [28] are optimal for all path-symmetric pure states in the absence of noises. Below, we apply these three types of measurements to the above phase resolution problem and investigate the accessible sensitivities with these measurements. We utilize a double-port measurement strategy, in which the photons at each output port of the interferometer are simultaneously detected. The reason is that the double-port measurement scheme may extract more information than the single-port one (see Appendix A for a demonstration of this superiority).

\section{A. Parity measurement}

Parity measurement was originally proposed to probe atomic frequency in trapped ions by Bollinger et al. [42] and later employed for optical interferometry by Gerry [43]. It accounts for distinguishing the states with even and odd numbers of photons. Specifically, the parity is assigned as the value of +1 when the photon number of a state is even and the value of -1 when odd. A parity operator acting on the output mode $c$ can be described by $[24,44,45]$

$$
\hat{\Pi}_{c}=(-1)^{n_{c}}=\exp \left[i \pi\left(\hat{J}_{0}+\hat{J}_{z}\right)\right] .
$$

Analogously, a parity operator acting on the output mode $d$ is defined by

$$
\hat{\Pi}_{d}=(-1)^{n_{d}}=\exp \left[i \pi\left(\hat{J}_{0}-\hat{J}_{z}\right)\right] .
$$

Obviously, they satisfy $\hat{\Pi}_{i}^{2}=\mathbb{1}(i=c, d)$, with $\mathbb{1}$ the identity matrix. To facilitate our calculation below, here we consider the operation of the second BS as a part of the measurement. Then the parity measurement on each output port through the BS is transformed into [24, 44, 45]

$$
\begin{aligned}
& \hat{\pi}_{c}=\hat{B} \hat{\Pi}_{c} \hat{B}^{\dagger}=\sum_{N=0}^{\infty} \sum_{k=0}^{N}|k, N-k\rangle\langle N-k, k|, \\
& \hat{\pi}_{d}=\hat{B} \hat{\Pi}_{d} \hat{B}^{\dagger}=\sum_{N=0}^{\infty} \sum_{k=0}^{N}(-1)^{N}|k, N-k\rangle\langle N-k, k| .
\end{aligned}
$$

Also, we have

$$
\hat{\pi}_{c} \hat{\pi}_{d}=\hat{\Pi}_{c} \hat{\Pi}_{d}=\sum_{N=0}^{\infty} \sum_{k=0}^{N}(-1)^{N}|k, N-k\rangle\langle k, N-k|,
$$

as a result of the commutation of $\left[\hat{J}_{0}, \hat{B}\right]=0$. From above expressions, we see that both $\hat{\pi}_{c}$ and $\hat{\pi}_{d}$ are of antidiagonal form, but slightly different up to a term $(-1)^{N}$, and $\hat{\pi}_{c} \hat{\pi}_{d}$ is of diagonal form. Note that the nonzero elements of $\hat{\pi}_{c}$ are all equal to 1 and those for $\hat{\pi}_{d}$ and $\hat{\pi}_{c} \hat{\pi}_{d}$ are assigned alternatively 1 and -1 .

The SP measurement scheme has been extensively used to probe the phase shift of the optical interferometer [24, 40, 43, 45-53]. In contrast, we apply here a doubleparity (DP) measurement scheme, in which we simultaneously perform parity detection on each output port of 
the interferometer. Formally, it can be expressed as a projection operator $\hat{M}_{\mathrm{P}}=\left\{\left|\mathrm{p}_{c}, \mathrm{p}_{d}\right\rangle\left\langle\mathrm{p}_{c}, \mathrm{p}_{d}\right|\right\}_{\mathrm{p}_{c}, \mathrm{p}_{d}= \pm 1}$, with $\left(\mathrm{p}_{c}, \mathrm{p}_{d}\right)$ the results of the parities at two output ports. We assume the second BS differs from the first one up to a $\pi$ phase factor. The conditional probability with respect to $\left(\mathrm{p}_{c}, \mathrm{p}_{d}\right)$ is defined by

$$
p\left(\mathrm{p}_{c}, \mathrm{p}_{d} \mid \phi\right)=\left\langle\mathrm{p}_{c}, \mathrm{p}_{d}\left|\hat{B}^{\dagger} \rho(\phi) \hat{B}\right| \mathrm{p}_{c}, \mathrm{p}_{d}\right\rangle,
$$

where we have employed the cyclic property of the trace operation. Using Eqs. (20) and (26) yields the CFI with respect to the DP measurement.

To calculate exactly the CFI, we further express the $F_{C}$ into an alternative form of Eq. (B6), as shown in Appendix B, which depends on three expectation values $\left\langle\hat{\Pi}_{c}\right\rangle,\left\langle\hat{\Pi}_{d}\right\rangle$ and $\left\langle\hat{\Pi}_{c} \hat{\Pi}_{d}\right\rangle$. With Eqs. (11) and (23)-(25), we obtain

$$
\begin{aligned}
\left\langle\hat{\Pi}_{c}\right\rangle & =D+E \cos \Delta \phi, \\
\left\langle\hat{\Pi}_{d}\right\rangle & =D+E(-1)^{\Delta} \cos \Delta \phi, \\
\left\langle\hat{\Pi}_{c} \hat{\Pi}_{d}\right\rangle & =\frac{1}{2}\left[\left(1-2 \eta_{a}\right)^{m}\left(1-2 \eta_{b}\right)^{n}+\left(1-2 \eta_{a}\right)^{n}\left(1-2 \eta_{b}\right)^{m}\right],
\end{aligned}
$$

where

$$
\begin{aligned}
& D=\frac{1}{2}{ }_{2} F_{1}\left(-m,-n ; 1 ; \frac{\eta_{a} \eta_{b}}{\gamma_{a} \gamma_{b}}\right)\left(\gamma_{a}^{m} \gamma_{b}^{n}+\gamma_{a}^{n} \gamma_{b}^{m}\right) \\
& E=\left(\begin{array}{c}
m \\
\Delta
\end{array}\right){ }_{2} F_{1}\left(-n,-n ; 1+\Delta ; \frac{\eta_{a} \eta_{b}}{\gamma_{a} \gamma_{b}}\right)\left(\eta_{a}^{\Delta / 2} \eta_{b}^{\Delta / 2} \gamma_{a}^{n} \gamma_{b}^{n}\right),
\end{aligned}
$$

with $\gamma_{i} \equiv 1-\eta_{i}(i=a, b)$ and ${ }_{2} F_{1}(a, b ; c ; z)$ the ordinary hyper-geometric function [24]. If photon losses only occur on mode $a$, the above terms $D$ and $E$ can be simplified as

$$
D=\frac{1}{2}\left(\begin{array}{c}
m \\
n
\end{array}\right) \eta_{a}^{n} \gamma_{a}^{\Delta}, \quad E=\eta_{a}^{\Delta / 2+n}
$$

and $\left\langle\hat{\Pi}_{a} \hat{\Pi}_{b}\right\rangle$ becomes

$$
\left\langle\hat{\Pi}_{c} \hat{\Pi}_{d}\right\rangle=\frac{(-1)^{\Delta}}{2}\left[\left(2 \eta_{a}-1\right)^{m}+\left(2 \eta_{a}-1\right)^{n}\right] .
$$

We plot in Fig. 3 the CFI for the single- and doubleparity measurements as a function of $\phi$ for $|6:: 0\rangle$ and $|10:: 4\rangle$. In the ideal case, i.e., $\eta=1$, the CFI for both measurements merges and is equal to the QFI over the whole phase interval [40]. In the lossy cases, the CFI is dependent on $\phi$, in the manner of an oscillation with a period of $\pi / \Delta$. A remarkable finding is that the DP measurement is slightly superior to the SP measurement in these cases. Similar results also take place in the case of losses occurring in one arm. The difference between the two measurements can be reasonably explained as follows. In the lossless cases, the probe states of consideration are pure states of definite photon number. This leads to a metrological equivalence between the SP and DP measurements. The reason is that the value of the parity on one output mode completely depends on the parity value from the other output mode, and thus adding one more parity measurement leads to no additional benefit to the SP measurement. However, the situation becomes different when losses occur. In the lossy cases, the probe states become mixed as Eq. (11) featuring fluctuation of the photon number. Then the results of parity measurements on the two output ports are independent such that by using the DP measurement can acquire more information comparing against the use of the SP measurement. In other words, the benefit of the DP comes from the fluctuation of the photon number (see Appendix $\mathrm{C}$ for a detailed comparison between the two-parity measurement schemes).

From Figs. 3(a) and 3(b) we see that the decreasing rate of the CFI for $|10:: 4\rangle$ is faster than that for $|6:: 0\rangle$. This can be seen more clearly in Fig. 2, where the phase sensitivities with the DP measurement are plotted as a function of $\eta$ by setting $\phi=\pi / 2 \Delta$. Such a faster deterioration accounts for the disadvantage of PEFSs over NOON states in the presence of photon losses, as also shown in Ref. [24]. Hence, although an additional enhancement of sensitivity may be achievable by the DP measurement compared with the single one, such an enhancement fails to reflect the robustness of the PEFS strategy compared with the NOON state one.

\section{B. Photon-number-resolving measurement}

A Photon-number-resolving (DPNR) measurement can be represented as the projection operator $\hat{M}_{\mathrm{N}}=$ $\left\{\left|n_{c}, n_{d}\right\rangle\left\langle n_{c}, n_{d}\right|\right\}_{n_{c}, n_{d}=0}^{\infty}[25,27,41,54]$, where the pairs of outcomes $\left(n_{c}, n_{d}\right)$ are the photon numbers detected at the $c$ and $d$ output ports of the interferometer. It has been shown that such a measurement is globally optimal to saturate the quantum Cramér-Rao bound for all pathsymmetric pure states in the absence of photon losses $[26,27]$. To evaluate the CFI with respect to the DPNR measurement, we should determine the conditional probability in terms of $\left(n_{c}, n_{d}\right)$ as

$$
p\left(n_{c}, n_{d} \mid \phi\right)=\left\langle n_{c}, n_{d}\left|B \rho(\phi) B^{\dagger}\right| n_{c}, n_{d}\right\rangle
$$

The analytical evaluation of the CFI for PEFSs is computationally involved (except for NOON states). We thus employ numerical calculations for the CFI.

As mentioned in Sec. II, the lossy NOON state is given by Eq. (14). By identifying $2 j=n_{c}+n_{d}$ and $2 \mu=n_{c}-n_{d}$, we have $p\left(n_{c}, n_{d} \mid \phi\right)=p(j, \mu \mid \phi)$, and then obtain 


$$
p(j, \mu \mid \phi)=\sqrt{\eta_{a}^{N} \eta_{b}^{N}}\left[d_{\mu, \frac{N}{2}}^{j}\left(\frac{\pi}{2}\right)\right]^{2}(-1)^{j+\mu} \cos (N \phi) \delta_{j, \frac{N}{2}}+\frac{1}{2} \sum_{k=0}^{N}\left[d_{\mu, \frac{N-k}{2}}^{j}\left(\frac{\pi}{2}\right)\right]^{2}\left(\begin{array}{l}
N \\
k
\end{array}\right)\left(\eta_{a}^{N-k} \gamma_{a}^{k}+\eta_{b}^{N-k} \gamma_{b}^{k}\right) \delta_{j, \frac{N-k}{2}},
$$

where $d_{\mu, \nu}^{j}(\beta)=\langle j, \mu|B| j, \nu\rangle$ refers to the Wigner rotation function and $\delta_{i, j}$ denotes the Kronecker delta function. Substituting the result of Eq. (35) into Eq. (20) and then doing the cancellation of terms in terms of $j \neq N / 2$ as a consequence of the property of $\delta_{i, j}$, finally yields

$$
F_{C}^{\mathrm{NOON}}=g\left(\eta_{a}, \eta_{b}\right) F_{Q}^{\mathrm{NOON}}
$$

with

$$
g\left(\eta_{a}, \eta_{b}\right)=\frac{\left(\eta_{a}^{N}+\eta_{b}^{N}\right) \sin ^{2}(N \phi)}{\left(\eta_{a}^{N}+\eta_{b}^{N}\right)-4 \frac{\eta_{a}^{N} \eta_{b}^{N}}{\eta_{a}^{N}+\eta_{b}^{N}} \cos ^{2}(N \phi)} .
$$

In the above derivation, we used the identity [27]

$$
\sum_{k=\text { even }}\left[d_{k-j, \frac{N}{2}}^{\frac{N}{2}}\left(\frac{\pi}{2}\right)\right]^{2}=\sum_{k=\text { odd }}\left[d_{k-j, \frac{N}{2}}^{\frac{N}{2}}\left(\frac{\pi}{2}\right)\right]^{2}=\frac{1}{2} .
$$

Equation (36) suggests that the DPNR measurement serves as the optimal measurement when $g\left(\eta_{a}, \eta_{b}\right)=1$. This equality holds, according to Eq. (37), when either of the following two conditions is satisfied: (a) $\eta_{a}=\eta_{b}$; or (b) $\phi \rightarrow \pi / 2 N$. In particular, for the condition $\eta_{a}=\eta_{b}$, the equality of $F_{C}^{\mathrm{NOON}}=F_{Q}^{\mathrm{NOON}}$ always holds independently of $\phi$. This means that the DPNR measurement is globally optimal over the whole range of values of phase parameter for equal loss rates of two arms. Additionally, as for PEFSs, we numerically evaluate the maximum sensitivity over $\phi$ with the DPNR measurement in the presence of photon losses.

As shown in Fig. (2), we numerically plot the phase sensitivities achievable with the DPNR measurement for $|6:: 0\rangle$ and $|10:: 4\rangle$, respectively. Similar to the parity measurements, the photon-number-resolving measurement also saturates the sensitivity bounds for the two probe states in the lossless case, i.e., $\eta=1$. This is an expected result, as demonstrated in previous works [26, 27, 41]. Our numerical evidence shows that the sensitivity bounds for $|6:: 0\rangle$ is indeed reachable with the DPNR measurement for both single- and two-arm losses, as analytically predicted above. From Fig. (2), although the DPNR measurement fails to be optimal for $|10:: 4\rangle$, it is more effective than the parity measurement; in particular, in the case of losses on one arm, it is nearly optimal for $|10:: 4\rangle$. More importantly, by employing the DPNR measurement, one can observe the loss resilience of the PEFS scheme over the NOON state one in lossy interferometry.

\section{Homodyne measurement}

A double-homodyne (DH) measurement can be represented as the projection operator $\hat{\boldsymbol{M}}_{\mathrm{H}}=|x, p\rangle\langle x, p|$ with a pair of outcomes $(x, p)$ corresponding to the eigenvalues of quadrature operators $\hat{X}$ and $\hat{P}$. Such a measurement scheme was initially applied to quantum teleportation of continuous variables $[55,56]$. Subsequently, Vidrighin et $a l$. found that this measurement also serves as the optimal measurement in the interferometric phase measurement with path-symmetric pure states in the absence of photon losses [28]. Our work also partially complements this literature, discussing the measurement in lossy cases.

To view its performance in our case, we need to calculate the CFI with respect to $\hat{M}_{\mathrm{H}}$. Because the results $x$ and $p$ of $\hat{\boldsymbol{M}}_{\mathrm{H}}$ are continuous variables, the CFI of Eq. (20) should be expressed in the integral form,

$$
F_{C}=\int_{-\infty}^{\infty} d x \int_{-\infty}^{\infty} d p \frac{1}{p(x, p \mid \phi)}\left(\frac{d p(x, p \mid \phi)}{d \phi}\right)^{2}
$$

where the conditional probability in terms of $(x, p)$ is given by

$$
p(x, p \mid \phi)=\left\langle n_{c}, n_{d}\left|B \rho(\phi) B^{\dagger}\right| n_{c}, n_{d}\right\rangle .
$$

For the sake of our calculation, we view the projector $\hat{M}_{\mathrm{H}}$ before the second BS. Each basis $|x, p\rangle$ hence corresponds to $|v(x, p)\rangle \equiv B^{\dagger}|x, p\rangle$. With the help of a discrete formulation of continuous variables $[28,57]$, we express the transformed basis $|v(x, p)\rangle$, by setting $\alpha=x+i p$, as

$$
|v(x, p)\rangle=\sum_{k, l} g_{k, l}(\alpha)|k, l\rangle,
$$

where

$g_{k, l}(\alpha)= \begin{cases}\frac{e^{-|\alpha|^{2} / 2}}{\sqrt{\pi}} \sqrt{\frac{l !}{k !}} \alpha^{k-l} L_{l}^{(k-l)}\left(|\alpha|^{2}\right), & k \geq l, \\ \frac{e^{-|\alpha|^{2} / 2}}{\sqrt{\pi}} \sqrt{\frac{k !}{l !}}\left(-\alpha^{*}\right)^{l-k} L_{k}^{(l-k)}\left(|\alpha|^{2}\right), & k<l,\end{cases}$

with $L_{n}^{(\Delta)}(x)$ denoting the generalized Laguerre polynomials. By alternatively expressing $\alpha=r e^{i \varphi}$, the above expression can be rewritten by

$$
g_{m, n}(r, \varphi)=g_{m, n}(r) e^{i(m-n) \varphi},
$$

with

$$
g_{k, l}(r)= \begin{cases}\frac{e^{-r^{2} / 2}}{\sqrt{\pi}} \sqrt{\frac{l !}{k !}} r^{k-l} L_{l}^{(k-l)}\left(r^{2}\right), & m \geq n \\ \frac{e^{-r^{2} / 2}}{\sqrt{\pi}} \sqrt{\frac{k !}{l !}}(-r)^{l-k} L_{k}^{(l-k)}\left(r^{2}\right), & m<n\end{cases}
$$

satisfying the relation $g_{l, k}(r)=g_{k, l}(r)(-1)^{k-l}$. With Eq. (11) we obtain the conditional probability in terms of $r$ and $\varphi$ for PEFSs as

$$
\begin{aligned}
p(r, \varphi \mid \phi)= & \sum_{k=0}^{m} \sum_{l=0}^{n}\left(d_{1}+d_{2}\right) g_{k, l}^{2}(r)+2 \cos [\Delta(\phi-2 \varphi)] \\
& \times \sum_{r=0}^{n} \sum_{r^{\prime}=0}^{n} d_{3} g_{r, \Delta+r^{\prime}}(r) g_{r^{\prime}, \Delta+r}(r)(-1)^{r-r^{\prime}-\Delta} .
\end{aligned}
$$


Transforming from Cartesian coordinates $(x, p)$ to polar coordinates $(r, \varphi)$, the CFI of Eq. (39) becomes

$$
F_{C}=\int_{0}^{\infty} r d r \int_{0}^{2 \pi} d \varphi \frac{1}{p(r, \varphi \mid \phi)}\left(\frac{d p(r, \varphi \mid \phi)}{d \phi}\right)^{2} .
$$

Submitting Eq. (45) into Eq. (46) finally yields the CFI with respect to the DH measurement. For NOON states, Eq. (45) can be simplified as

$$
\begin{aligned}
p(r, \varphi \mid \phi)= & \frac{1}{2} \sum_{l=0}^{N}\left(\begin{array}{c}
N \\
l
\end{array}\right) g_{N-l, 0}^{2}(r)\left[\eta_{a}^{N}\left(\eta_{a}^{-1}-1\right)^{l}+\eta_{b}^{N}\left(\eta_{b}^{-1}-1\right)^{l}\right] \\
& +(-1)^{N} \sqrt{\eta_{a}^{N} \eta_{b}^{N}} g_{N, 0}^{2}(r) \cos [N(\phi-2 \varphi)] .(47)
\end{aligned}
$$

For simplicity, we impose the restriction that the two arms have equal photon loss rates (i.e., $\eta_{1}=\eta_{2}=\eta$ ).

In Fig. 3, we plot the phase sensitivities achievable with the DH measurement compared against the DP measurement. Unlike the DP measurement, the sensitivities with the DH measurement do not vary with $\phi$. It is as clear as the fact that the value of the integral in Eq. (46) remains unchanged by replacing $\varphi$ with $\varphi+\phi / 2$ in the way that Eqs. (45) and (47) become $\phi$-independent. What is unexpectation is that the $\mathrm{DH}$ measurement exhibits poorer performance for phase sensitivity in comparison to the DP measurement. As shown in Fig. 3, a slight noise causes the sensitivities attained with the DH measurement to decrease more significantly than that with the DP measurement. This result is seemly contradictory to previous findings that the homodyne detection was identified as a nearly optimal measurement scheme in a noisy Mach-Zehnder interferometer with coherent and squeezed vacuum light and far superior to the parity detection [58]. A reasonable explanation for this contradictory result is that the probe states of consideration here are non-Gaussian; in such cases the non-Gaussian measurements (i.e., parity detection and photon-numberresolving detection) are desirable over the Gaussian measurements (i.e., homodyne detection). Hence, the DH measurement still fails to reflect the robustness of the PEFS strategy compared with the NOON state one.

\section{Conclusion}

We have investigated in this paper the phase sensitivities of robust quantum optical interferometry with PEFSs proposed by Huver et al. [19] with the help of the quantum estimation theory. We analytically derived the QFI for PEFSs and NOON states in the presence of photon loss and quantitatively demonstrated that the PEFS strategy shows more robustness to photon loss than the NOON state strategy, which was first illustrated by $\mathrm{Hu}-$ ver et al based on a measurement which was yet to be implemented in experiments.

We then addressed how to implement this advantage with three feasible measurements, namely, parity, photon-number-resolving, and homodyne measurement.
Unlike the SP measurement scheme employed in Ref. [24], we alternatively applied a DP measurement scheme. We found that it can provide an additional enhancement in phase sensitivities in comparison to the SP measurement, but it still fails to observe the robustness of the PEFS strategy. Furthermore, we considered the DPNR measurement and found the DPNR measurement [59] to observe the loss resilience of the PEFS strategy over the NOON state one in the presence of loss. Interestingly, this type of measurement can always saturate the sensitivity bounds for lossy NOON states. Finally, we assessed the performance of the $\mathrm{DH}$ measurement in the aforementioned scenario. Our results show that the DH measurement has lower performance than the other two measurements.

To conclude, apart from the DPNR measurement, both the DP and DH measurements fail to indicate the robustness of the PEFSs to loss over the NOON states. A hierarchy for the performance of the measurements of consideration is given by $\hat{M}_{\mathrm{H}}<\hat{M}_{\mathrm{P}}<\hat{M}_{\mathrm{N}}$ when employing the PEFSs for lossy interferometry. Our results are readily applicable to other robust quantum metrological strategies [14, 20, 21, 23, 60, 61], and may shine some light on the possible detection schemes for noisy metrology.

Finally, the primary focus of this paper is to demonstrate the robustness of the metrological scheme with PEFSs by taking NOON states as a benchmark, following the approach used by Huver et al [19]. An alternative way to evaluate the robustness of a metrological scheme, as commonly used in a broad range of applications [15, 16, 59, 62-69], is to use the separable probe state as a benchmark. Although doing so may be beneficial to determine when both the PEFS and NOON state schemes lose their quantum-enhanced advantage in the presence of noises, we do not make a comparison to the separable state to stick to the topic of the paper. Our results also shed some light on this. It was shown in Fig. 1 that the sensitivities for both states are quite low at a low level of loss strength, but degrade increasingly as the loss strength increases. In other words, both states will lose their quantum advantage as the loss strength increases. However, what is different is the speed of degradation. Our results indicate that the sensitivity attained by the DPNR measurement for the PEFS degrades more slowly than that for the NOON state, which meets the theoretically predicted performance suggested by the QFI; However, it is just the reverse for both DP and DH measurements. These results reasonably answer the problem raised in Introduction.

\section{Acknowledgments}

We are grateful to Stefan Ataman for helpful suggestions and remarks and to the two anonymous referees for their enlightening comments and suggestions for our paper. This work was supported by the NSFC through 
Grants No. 12005106 and No. 11974189, the Natural Science Foundation of the Jiangsu Higher Education Institutions of China under Grant No. 20KJB140001, and a project funded by the Priority Academic Program Development of Jiangsu Higher Education Institutions. L.Z. acknowledges support from the China Postdoctoral Science Foundation under Grant No. 2018M642293.

\section{Appendix A: The superiority of the double-port measurement scheme over the single-port measurement scheme}

In this appendix, we demonstrate that the double-port measurement scheme is superior to the single-port measurement one. Here, the double-port measurement refers to detecting the states of photons at two output ports of the interferometer simultaneously and the single-port measurement refers to detecting the states of photons at one of the two output ports. Assuming that the outcome of a double-port measurement is represented by a variable pair $\left(\chi_{c}, \chi_{d}\right)$ of the detected probability denoted by $p\left(\chi_{c}, \chi_{d} \mid \phi\right)$, then the marginal probability of a single outcome is determined by

$$
p\left(\chi_{c} \mid \phi\right)=£ p\left(\chi_{c}, \chi_{d} \mid \phi\right) d \chi_{d}
$$

Having Eqs. (20) and (A1), we identify the relationship between the CFIs for the single- and double-port measurement schemes as

$$
\begin{aligned}
F_{C}^{\mathrm{S}} & =£ \frac{1}{p\left(\chi_{c} \mid \phi\right)}\left(\frac{d p\left(\chi_{c} \mid \phi\right)}{d \phi}\right)^{2} d \chi_{c} \\
& =£ \frac{1}{£ p\left(\chi_{c}, \chi_{d} \mid \phi\right) d \chi_{d}}\left(£ \frac{d p\left(\chi_{c}, \chi_{d} \mid \phi\right)}{d \varphi} d \chi_{d}\right)^{2} d \chi_{c} \\
& \leq £ \frac{1}{p\left(\chi_{c}, \chi_{d} \mid \phi\right)}\left(\frac{d p\left(\chi_{c}, \chi_{d} \mid \phi\right)}{d \phi}\right)^{2} d \chi_{c} d \chi_{d} \\
& =F_{C}^{\mathrm{D}},
\end{aligned}
$$

where the above inequality comes from the use of the Cauchy-Schwarz inequality and its equality holds if and only if

$$
\sqrt{p\left(\chi_{c}, \chi_{d} \mid \phi\right)}=\frac{\lambda}{\sqrt{p\left(\chi_{c}, \chi_{d} \mid \phi\right)}} \frac{d p\left(\chi_{c}, \chi_{d} \mid \phi\right)}{d \varphi}
$$

is satisfied with a nonzero number $\lambda$. The above condition is generally satisfied at some specific points of the phase or for some typical cases (see Appendix $\mathrm{C}$ for an example). However, it does not hold in most cases, in which we have $F_{C}^{\mathrm{D}}>F_{C}^{\mathrm{S}}$, such that the double-port measurement scheme is superior to the single-port measurement scheme in interferometric phase measurements.
Appendix B: A theoretical framework for the double parity measurement

In the following we propose a general theoretical framework for analysis of phase sensitivity with the DP measurement. As mentioned in the main text, the SP detection has two outcomes: +1 and -1 . For simplicity, here we denote them by + and - , respectively. Thus, the DP detection results in four outcomes:,,+++--+ , and -- . According to Eq. (20), the CFI with the DP measurement can be expanded to four terms as

$$
\begin{aligned}
F_{C}^{\mathrm{D}}= & \frac{\left[\partial_{\phi} P(++\mid \phi)\right]^{2}}{P(++\mid \phi)}+\frac{\left[\partial_{\phi} P(+-\mid \phi)\right]^{2}}{P(+-\mid \phi)} \\
& +\frac{\left[\partial_{\phi} P(-+\mid \phi)\right]^{2}}{P(-+\mid \phi)}+\frac{\left[\partial_{\phi} P(--\mid \phi)\right]^{2}}{P(--\mid \phi)} .
\end{aligned}
$$

We find that the conditional probabilities in terms of the four outcomes satisfy the equations

$P(++\mid \phi)+P(+-\mid \phi)+P(-+\mid \phi)+P(--\mid \phi)=1$,

$P(++\mid \phi)+P(+-\mid \phi)-P(-+\mid \phi)-P(--\mid \phi)=\left\langle\Pi_{c}\right\rangle$,

$P(++\mid \phi)+P(-+\mid \phi)-P(+-\mid \phi)-P(--\mid \phi)=\left\langle\Pi_{d}\right\rangle$,

$P(++\mid \phi)+P(--\mid \phi)-P(+-\mid \phi)-P(-+\mid \phi)=\left\langle\Pi_{c} \Pi_{d}\right\rangle$.

One can reverse these equations and solve for the conditional probabilities in terms of the expectation values $\left\langle\Pi_{c}\right\rangle,\left\langle\Pi_{d}\right\rangle$, and $\left\langle\Pi_{c} \Pi_{d}\right\rangle$. Finally, substituting them into Eq. (B1) yields

$$
\begin{aligned}
F_{C}^{\mathrm{D}}= & \frac{1}{2} \frac{\left(1+\left\langle\Pi_{c} \Pi_{d}\right\rangle\right)\left[\partial_{\phi}\left(\left\langle\Pi_{c}\right\rangle+\left\langle\Pi_{d}\right\rangle\right)\right]^{2}}{\left(1+\left\langle\Pi_{c} \Pi_{d}\right\rangle\right)^{2}-\left(\left\langle\Pi_{c}\right\rangle+\left\langle\Pi_{d}\right\rangle\right)^{2}} \\
& +\frac{1}{2} \frac{\left(1-\left\langle\Pi_{c} \Pi_{d}\right\rangle\right)\left[\partial_{\phi}\left(\left\langle\Pi_{c}\right\rangle-\left\langle\Pi_{d}\right\rangle\right)\right]^{2}}{\left(1-\left\langle\Pi_{c} \Pi_{d}\right\rangle\right)^{2}-\left(\left\langle\Pi_{c}\right\rangle-\left\langle\Pi_{d}\right\rangle\right)^{2}} .
\end{aligned}
$$

Defining the operators $\hat{\Pi}_{ \pm} \equiv \hat{\Pi}_{c} \pm \hat{\Pi}_{d}$ and associating them with $\Pi_{ \pm}^{2}=2\left(1 \pm \Pi_{c} \Pi_{d}\right)$, we can rewrite Eq. (B6) in a concise form as

$$
F_{C}^{\mathrm{D}}=\sum_{i= \pm} \frac{\left\langle\hat{\Pi}_{i}^{2}\right\rangle\left(\partial_{\phi}\left\langle\hat{\Pi}_{i}\right\rangle\right)^{2}}{\frac{1}{4}\left\langle\hat{\Pi}_{i}^{2}\right\rangle^{2}-\left\langle\hat{\Pi}_{i}\right\rangle^{2}} .
$$

The expression (B6) is generally divided into two parts, based on whether the photon number of states is even or odd. The first term in Eq. (B6) is contributed by states grouped in the subspace of an even photon number and the second term is contributed by those of an odd photon number.

\section{Appendix C: Comparison between the single and double parity measurements}

We wish to give more insight into Eq. (B6). When the total photon number of the system, denoted by $N$, 


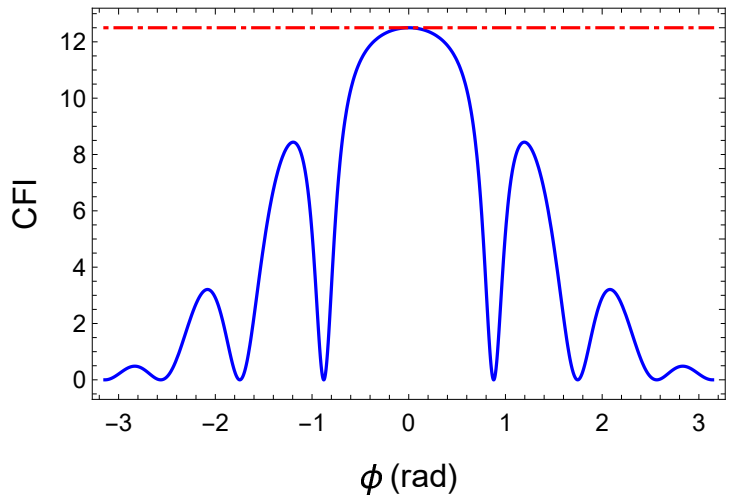

Figure 4. (Color online) Classical Fisher information as a function of $\phi$ for $(|3:: 0\rangle+|4:: 0\rangle) / \sqrt{2}$ with the DP and SP measurements. The dash-dotted red line corresponds to the DP measurement and the solid blue curve to the SP measurement.

is fixed, Eq. (B6) can be simplified into that of the SP measurement [40]

$$
F_{C}^{S}=\frac{\left[\partial_{\phi}\left\langle\Pi_{c}\right\rangle\right]^{2}}{1-\left\langle\Pi_{c}\right\rangle^{2}}
$$

as a result of the fact that, for even $N$, we have $\left\langle\Pi_{c}\right\rangle=$ $\left\langle\Pi_{d}\right\rangle$ and $\left\langle\Pi_{c} \Pi_{d}\right\rangle=1$, and for odd $N$, we have $\left\langle\Pi_{c}\right\rangle=$ $-\left\langle\Pi_{d}\right\rangle$ and $\left\langle\Pi_{c} \Pi_{d}\right\rangle=-1$. This implies that the DP measurement is metrologically equivalent to the SP measurement when the number of total photons is fixed. This equivalence can be well understood. For probe states with a fixed photon number, the value of the parity on one output port of the interferometer is completely determined by the parity value from the other output port; then adding one more parity detection leads to no additional benefit. A classic example is the NOON states, for which both the SP and DP measurements are identified as the optimal measurement, in the manner of saturating the Heisenberg-scaling sensitivity over the entire phase range [40].

When the fluctuation of the photon number exists, the DP and SP measurements may not be equivalent, except at some specific phases. In order to elucidate this, we choose a superposition between two NOON states of even and odd photon numbers as the probe state

$$
|\psi\rangle=\sqrt{p}\left|N_{\mathrm{e}}:: 0\right\rangle+\sqrt{1-p}\left|N_{\mathrm{o}}:: 0\right\rangle .
$$

Obviously, this type of probe state has fluctuation of the photon number. According to Eqs. (23)-(25), we explicitly obtain the expectations with respect to Eq. (C2) as

$$
\begin{aligned}
\left\langle\Pi_{c}\right\rangle & =p \cos \left(N_{\mathrm{e}} \phi\right)+(1-p) \cos \left(N_{\mathrm{o}} \phi\right), \\
\left\langle\Pi_{d}\right\rangle & =p \cos \left(N_{\mathrm{e}} \phi\right)-(1-p) \cos \left(N_{\mathrm{o}} \phi\right), \\
\left\langle\Pi_{c} \Pi_{d}\right\rangle & =2 p-1 .
\end{aligned}
$$

Substituting these results into Eqs. (B6) and (C1) then gives

$$
\begin{aligned}
& F_{C}^{\mathrm{D}}=p N_{\mathrm{e}}^{2}+(1-p) N_{\mathrm{o}}^{2}, \\
& F_{C}^{\mathrm{S}}=\frac{\left[p N_{\mathrm{e}} \sin \left(N_{\mathrm{e}} \phi\right)+(1-p) N_{\mathrm{o}} \sin \left(N_{\mathrm{o}} \phi\right)\right]^{2}}{1-\left[p \cos \left(N_{\mathrm{e}} \phi\right)-(1-p) \cos \left(N_{\mathrm{o}} \phi\right)\right]^{2}} .
\end{aligned}
$$

Interestingly, we can find that $F_{C}^{\mathrm{D}}$ is identical to $F_{Q}$ for the state of Eq. (C2), meaning that the DP measurement is an optimal measurement in the manner of $\phi$ independence. Note that a phase-averaged operation is required in the calculation of the QFI, since an external reference beam is absent in the parity detection [37]. Eqs. (C6) and $(\mathrm{C} 7)$ are plotted in Fig. 4 for the case where $p=1 / 2$, $N_{\mathrm{o}}=3$, and $N_{\mathrm{e}}=4$, i.e., the equal superposition of threeand four-photon NOON states. It can been seen that the value of $F_{C}^{\mathrm{S}}$ is lower than that of $F_{C}^{\mathrm{D}}$ over the whole phase interval except at the zero-point location, where they have merged satisfactorily.
[1] H. Lee, P. Kok, and J. P. Dowling, J. Mod. Opt. 49, 2325 (2002).

[2] V. Giovannetti, S. Lloyd, and L. Maccone, Science 306, 1330 (2004).

[3] V. Giovannetti, S. Lloyd, and L. Maccone, Phys. Rev. Lett. 96, 010401 (2006).

[4] V. Giovannetti, S. Lloyd, and L. Maccone, Nat. Photon. 5, 222 (2011).

[5] C. L. Degen, F. Reinhard, and P. Cappellaro, Rev. Mod. Phys. 89, 035002 (2017).

[6] L. Pezzè, A. Smerzi, M. K. Oberthaler, R. Schmied, and P. Treutlein, Rev. Mod. Phys. 90, 035005 (2018).

[7] J. Liu, H. Yuan, X.-M. Lu, and X. Wang, J. Phys. A: Math. Theor. 53, 023001 (2019).

[8] E. Polino, M. Valeri, N. Spagnolo, and F. Sciarrino, AVS Quantum Sci. 2, 024703 (2020).

[9] J. G. Rarity, P. R. Tapster, E. Jakeman, T. Larchuk,
R. A. Campos, M. C. Teich, and B. E. A. Saleh, Phys. Rev. Lett. 65, 1348 (1990).

[10] P. Walther, J.-W. Pan, M. Aspelmeyer, R. Ursin, S. Gasparoni, and A. Zeilinger, Nature (London) 429, 158 (2004).

[11] M. W. Mitchell, J. S. Lundeen, and A. M. Steinberg, Nature (London) 429, 161 (2004).

[12] T. Nagata, R. Okamoto, J. L. Brien, K. Sasaki, and S. Takeuchi, Science 316, 726 (2007).

[13] G. Y. Xiang, B. L. Higgins, D. W. Berry, H. M. Wiseman, and G. J. Pryde, Nat. Photon. 5, 43 (2011).

[14] U. Dorner, R. Demkowicz-Dobrzanski, B. J. Smith, J. S. Lundeen, W. Wasilewski, K. Banaszek, and I. A. Walmsley, Phys. Rev. Lett. 102, 040403 (2009).

[15] B. M. Escher, R. L. de Matos Filho, and L. Davidovich, Nat. Phys. 7, 406 (2011).

[16] R. Demkowicz-Dobrzanski, J. Kolodynski, and M. Guta, 
Nat. Commun. 3, 1063 (2012).

[17] M. H. Michael, M. Silveri, R. T. Brierley, V. V. Albert, J. Salmilehto, L. Jiang, and S. M. Girvin, Phys. Rev. X 6, 031006 (2016).

[18] S. Zhou, M. Zhang, J. Preskill, and L. Jiang, Nat. Commun. 9, 78 (2018).

[19] S. D. Huver, C. F. Wildfeuer, and J. P. Dowling, Phys. Rev. A 78, 063828 (2008).

[20] T.-W. Lee, S. D. Huver, H. Lee, L. Kaplan, S. B. McCracken, C. Min, D. B. Uskov, C. F. Wildfeuer, G. Veronis, and J. P. Dowling, Phys. Rev. A 80, 063803 (2009).

[21] J. Kołodyński and R. Demkowicz-Dobrzański, Phys. Rev. A 82, 053804 (2010).

[22] R. Demkowicz-Dobrzanski, U. Dorner, B. J. Smith, J. S. Lundeen, W. Wasilewski, K. Banaszek, and I. A. Walmsley, Phys. Rev. A 80, 013825 (2009).

[23] S. Knysh, V. N. Smelyanskiy, and G. A. Durkin, Phys. Rev. A 83, 021804(R) (2011).

[24] K. Jiang, C. J. Brignac, Y. Weng, M. B. Kim, H. Lee, and J. P. Dowling, Phys. Rev. A 86, 013826 (2012).

[25] L. Pezzé and A. Smerzi, Phys. Rev. Lett. 100, 073601 (2008).

[26] H. F. Hofmann, Phys. Rev. A 79, 033822 (2009).

[27] W. Zhong, Y. Huang, X. Wang, and S. L. Zhu, Phys. Rev. A 95, 052304 (2017).

[28] M. D. Vidrighin, G. Donati, M. G. Genoni, X. M. Jin, W. S. Kolthammer, M. S. Kim, A. Datta, M. Barbieri, and I. A. Walmsley, Nat. Commun. 5, 3532 (2014).

[29] J. Rubio and J. Dunningham, New Journal of Physics 21, 043037 (2019).

[30] C. W. Helstrom, Quantum Detection and Estimation Theory (Academic, New York, 1976).

[31] A. S. Holevo, Probabilistic and Statistical Aspects of Quantum Theory (North-Holland, Amsterdam, 1982).

[32] S. L. Braunstein and C. M. Caves, Phys. Rev. Lett. 72, 3439 (1994).

[33] W. Zhong, X. M. Lu, X. X. Jing, and X. G. Wang, J. Phys. A: Math. Theor. 47, 385304 (2014).

[34] B. Yurke, S. L. McCall, and J. R. Klauder, Phys. Rev. A 33, 4033 (1986).

[35] A. Preda and S. Ataman, Phys. Rev. A 99, 053810 (2019).

[36] W. Zhong, F. Wang, L. Zhou, P. Xu, and Y. B. Sheng, Sci. China Phys. Mech. Astron. 63, 260312 (2020).

[37] M. Jarzyna and R. Demkowicz-Dobrzański, Phys. Rev. A 85, 011801(R) (2012).

[38] H. Uys and P. Meystre, Phys. Rev. A 76, 013804 (2007).

[39] R. Krischek, C. Schwemmer, W. Wieczorek, H. Weinfurter, P. Hyllus, L. Pezzé, and A. Smerzi, Phys. Rev. Lett. 107, 080504 (2011).

[40] K. P. Seshadreesan, S. Kim, J. P. Dowling, and H. Lee, Phys. Rev. A 87, 043833 (2013).

[41] M. D. Lang and C. M. Caves, Phys. Rev. Lett. 111, 173601 (2013).

[42] J. J. . Bollinger, W. M. Itano, D. J. Wineland, and D. J. Heinzen, Phys. Rev. A 54, R4649 (1996).

[43] C. C. Gerry, Phys. Rev. A 61, 043811 (2000).

[44] Y. Gao, P. M. Anisimov, C. F. Wildfeuer,
J. Luine, H. Lee, and J. P. Dowling, J. Opt. Soc. Am. B 27, A170 (2010).

[45] A. Chiruvelli and H. Lee, J. Mod. Opt. 58, 945 (2011).

[46] W. N. Plick, P. M. Anisimov, J. P. Dowling, H. Lee, and G. S. Agarwal, New J. Phys. 12, 113025 (2010).

[47] C. C. Gerry and J. Mimih, Contemp. Phys. 51, 497 (2010).

[48] Q. S. Tan, J. Q. Liao, X. G. Wang, and F. Nori, Phys. Rev. A 89, 053822 (2014).

[49] K. P. Seshadreesan, P. M. Anisimov, H. Lee, and J. P. Dowling, New J. Phys. 13, 083026 (2011).

[50] J. Joo, W. J. Munro, and T. P. Spiller, Phys. Rev. Lett. 107, 083601 (2011).

[51] R. A. Campos, C. C. Gerry, and A. Benmoussa, Phys. Rev. A 68, 023810 (2003).

[52] P. M. Anisimov, G. M. Raterman, A. Chiruvelli, W. N. Plick, S. D. Huver, H. Lee, and J. P. Dowling, Phys. Rev. Lett. 104, 103602 (2010).

[53] Z. Huang, K. R. Motes, P. M. Anisimov, J. P. Dowling, and D. W. Berry, Phys. Rev. A 95, 053837 (2017).

[54] L. Pezzé and A. Smerzi, Phys. Rev. Lett. 110, 163604 (2013).

[55] A. Furusawa, J. L. Sørensen, S. L. Braunstein, C. A. Fuchs, H. J. Kimble, and E. S. Polzik, Science 282, 706 (1998).

[56] S. L. Braunstein and H. J. Kimble, Phys. Rev. Lett. 80, 869 (1998).

[57] S. J. van Enk, Phys. Rev. A 60, 5095 (1999).

[58] B. T. Gard, C. You, D. K. Mishra, R. Singh, H. Lee, T. R. Corbitt, and J. P. Dowling, EPJ Quantum Technology 4, 4 (2017).

[59] S. F. Huelga, C. Macchiavello, T. Pellizzari, A. K. Ekert, M. B. Plenio, and J. I. Cirac, Phys. Rev. Lett. 79, 3865 (1997).

[60] S.-Y. Lee, C.-W. Lee, J. Lee, and H. Nha, Scientific Reports 6, 30306 (2016).

[61] G. S. Thekkadath, M. E. Mycroft, B. A. Bell, C. G. Wade, A. Eckstein, D. S. Phillips, R. B. Patel, A. Buraczewski, A. E. Lita, T. Gerrits, S. W. Nam, M. Stobińska, A. I. Lvovsky, and I. A. Walmsley, npj Quantum Information 6, 89 (2020).

[62] R. Chaves, L. Aolita, and A. Acín, Phys. Rev. A 86, 020301 (2012).

[63] A. W. Chin, S. F. Huelga, and M. B. Plenio, Phys. Rev. Lett. 109, 233601 (2012).

[64] R. Chaves, J. B. Brask, M. Markiewicz, J. Kołodyński, and A. Acín, Phys. Rev. Lett. 111, 120401 (2013).

[65] Fröwis, M. Skotiniotis, B. Kraus, and W. Dür, New Journal of Physics 16, 083010 (2014).

[66] J. B. Brask, R. Chaves, and J. Kołodyński, Phys. Rev. X 5, 031010 (2015).

[67] A. Smirne, J. Kołodyński, S. F. Huelga, and R. Demkowicz-Dobrzański, Phys. Rev. Lett. 116, 120801 (2016).

[68] S. P. Nolan, S. S. Szigeti, and S. A. Haine, Phys. Rev. Lett. 119, 193601 (2017).

[69] R.-J. Cai, W. Zhong, L. Zhou, and Y.-B. Sheng, Quantum Information Processing 19, 359 (2020). 